\title{
MicroRNAs in Human Malignant Gliomas
}

\section{Masahiro Mizoguchi, Yanlei Guan, Koji Yoshimoto, Nobuhiro Hata, Toshiyuki Amano, Akira Nakamizo, and Tomio Sasaki}

Department of Neurosurgery, Graduate School of Medical Sciences, Kyushu University, 3-1-1 Maidashi, Higashi-ku, Fukuoka 812-8582, Japan

Correspondence should be addressed to Masahiro Mizoguchi,mmizoguc@ns.med.kyushu-u.ac.jp

Received 16 March 2012; Revised 20 May 2012; Accepted 21 May 2012

Academic Editor: Arrigo De Benedetti

Copyright ( 2012 Masahiro Mizoguchi et al. This is an open access article distributed under the Creative Commons Attribution License, which permits unrestricted use, distribution, and reproduction in any medium, provided the original work is properly cited.

\begin{abstract}
MicroRNA (miRNA) is a new class of small noncoding RNA molecules that regulate a wide spectrum of gene expression in a posttranscriptional manner. MiRNAs play crucial roles in tumorigenesis, angiogenesis, invasion, and apoptosis for various types of tumor. Recent studies have identified dysregulation of specific miRNAs in malignant gliomas. Global expression profiling of miRNAs has revealed several miRNAs clinically implicated in human glioblastomas. Some miRNAs are clearly associated with clinical outcome and chemo- and radio-therapy resistance in these tumors. Furthermore, miRNAs also regulate specific signaling pathways, including the critical core pathways in glioblastoma. As a result, miRNAs have the potential to affect the responses to molecular-targeted therapies. More recent studies have revealed that miRNAs might be associated with cancer stem cell properties, affecting tumor maintenance and progression. Recent investigation have revealed that miRNAs are not only biological markers with diagnostic implications, but also one of the most promising treatment targets in human glioblastoma. Herein, we summarized the novel insights of miRNAs into human malignant gliomas.
\end{abstract}

\section{Introduction}

Glioblastoma is one of the most common and malignant primary brain tumors in adults. Glioblastoma is essentially a pathogenetically heterogeneous tumor, and resent large-scale genomic analyses have allowed molecular subclassification of glioblastomas [1-3].

Recent work has identified a class of small noncoding RNA molecules, named microRNA (miRNA), which regulate a wide spectrum of gene expression in a posttranscriptional manner [4, 5]. More than 1500 precursors and 1921 mature human miRNAs have been discovered and registered in miRBase to date [6], and these miRNAs reportedly regulate approximately $30 \%$ of all protein-coding genes [7]. At present, it is predicted that more than half of protein coding genes would be regulated by miRNAs. The incorporation of the mature miRNA into an effector complex, called RNAinduced silencing complex (RISC), binds to messenger RNA (mRNA) and can affect the translation and stability of mRNA [7]. Recent reports have revealed that miRNAs play crucial roles in tumorigenesis, angiogenesis, invasion, and apoptosis in various types of tumor $[4,5]$. In addition, previous miRNA expression profiling may yield more accurate classification of human cancers than mRNA expression profiling [8]. A recent large-scale multidimensional analysis of molecular characteristics, The Cancer Genome Atlas (TCGA), which includes the expression profiles of miRNA as well as DNA copy number, gene expression, and DNA methylation, has revealed frequent genetic alterations in three critical core pathways [9]. The present paper summarizes recent insights regarding miRNA and human malignant gliomas.

\section{Identification of MicroRNAs}

In 2002, Calin et al. first reported the frequent deletions and downregulation of miR-15 and miR-16 at chromosome $13 q 14$ in a majority of cases of chronic lymphocytic leukemia (CLL) [10] and also demonstrated that more than half of miRNA genes are located in cancer-associated genomic 
regions or in fragile sites [11]. In addition, Lu et al. reported that the classification of malignant tumors based on miRNA expression profiles was more precise than mRNA profiles [8]. These findings have facilitated miRNA research in the field of oncology. In 2005, two groups initially reported miRNA dysregulation in glioblastoma using microarray analysis $[12,13]$. Chan et al. identified five upregulated and three downregulated miRNAs out of 180 miRNAs in glioblastoma, while Ciafré et al. also demonstrated nine upregulated and four downregulated miRNAs. A common miRNA identified by both groups was miR-21, which was revealed to act as an antiapoptotic factor $[12,14]$. Both groups used microarray analysis to identify miRNAs, whose expression was altered in glioblastoma. Subsequently several studies by using microarray analysis have also reported. Godlesski et al. and Sasayama et al. reported additional miRNAs dysregulated in glioblastoma $[15,16]$. They also investigated the biological function of miR-128 and miR-10b, respectively $[15,16]$. We subsequently applied the stem-loop reverse transcriptionPCR array, which can evaluate miRNA expression with sensitivity and specificity superior to microarrays $[17,18]$. Our study identified 16 miRNAs for which expression was significantly altered in glioblastoma (WHO grade 4) compared with anaplastic astrocytoma (WHO grade 3). Furthermore, we clearly demonstrated that overexpression of miR-196a or $-196 \mathrm{~b}$ is correlated with shorter overall survival among patients with malignant glioma [19]. In the same year, Malzkorn et al. also identified 12 upregulated miRNAs involved in the malignant progression of gliomas using stem-loop real-time RT-PCR [20]. Kim et al. also reported clinical implications of miR-26 gene amplification in glioblastoma patients by using TCGA data [21]. Recently, Rao et al. performed locked nucleic acid (LNA) array for a large-scale, genomewide miRNA expression profile and identified 55 upregulated and 29 downregulated miRNAs in malignant gliomas [22]. More important thing of this study was that a cluster of only 23 miRNAs was sufficient to distinguish glioblastoma from anaplastic astrocytoma with an accuracy of 95\% [22]. Furthermore, Srinivasan et al. identified a ten-miRNA expression signature, which could predict overall survival of glioblastoma patients [23]. More recently, combination of mRNA and miRNA expression profiling signature identified five glioblastoma subclasses [24].

In this paper, miRNAs dysregulated, which were identified by recent global studies for glioblastomas, were summarized (Table 1 ). Each report has demonstrated several miRNAs, whose expression was significantly altered in glioblastoma compared with normal brain tissues or lowergrade gliomas. Taken together, 52 upregulated miRNAs and 33 downregulated miRNAs have been reported by seven global studies published between 2005-2010 (Table 1). Interestingly, the results of each report were quite varied. One of the causes of this diversity is the difference of methodology, platform, and control samples. Consequently novel miRNAs, of which more than three studies confirmed aberrations, were only four miRNAs (miR-21, miR-10b, miR-128-1, and miR-128-2). In fact, newly identified miRNAs have not been elucidated in previous analysis; however, the number of concordance among these studies is extremely small. It would be better to say that these four miRNAs may be consistently important in glioblastomas.

\section{3. $\mathrm{miR}-21$}

One of the most common miRNAs identified by recent studies of gliomas, miR-21 has been revealed to act as an antiapoptotic factor that targets a network of p53, transforming growth factor (TGF)- $\beta$, and mitochondrial apoptosis tumor suppressor genes in glioblastoma cells [12, 14]. Overexpressed miR-21 is a unique miRNA, whose overexpression is identified in a large number of cancers investigated to date. Essentially, Medina et al. first demonstrated that tumors addicted to oncomiRs by using an in vivo model of miR-21-induced pre-B-cell lymphoma [25]. This result has facilitated the research elucidating the potential application as therapeutic targets in cancer. Our data revealed that miR21 expression is significantly higher in glioblastoma than anaplastic astrocytoma (3.6 fold); however, the expression did not affect patients' outcome [19]. The important point to note is that miR-21 is only one miRNA, whose overexpression is identified in all of the recent reports. In addition, previous large study identified miR-21 upregulation in all of tumor types [26]. MiR-21 is considered to be one of the critical miRNA in tumorigenesis.

\section{4. $\mathrm{miR}-196$}

We have already reported a significant association between high expression of miR-196 and shorter overall survival among glioblastoma patients [19]. Expression of miR-196a and miR-196b is extremely high compared with other overexpressed miRNAs in glioblastoma, so both of these two miRNAs are considered to be associated with the malignant transformation of gliomas [19]. Overexpression of miR-196 has also recently been identified in several cancers [27]. Bloomston et al. first reported the clinical implications of miR-196a-2 in patients with pancreatic cancer [28]. Our data also showed correlations between overexpression of miR-196 and shorter overall survival with malignant gliomas [19]. The characteristic of miR-196 is the location in the vicinity of homeobox (HOX) clusters within the genome of vertebrate genomes [29]. Three miR-196 genes have been identified; miR-196a-1 located on chromosome 17 (17q21.32) at HOXB cluster, miR-196a-2 located on chromosome12 (12q13.13) at HOXC cluster, and miR-196b is located on chromosome 7 (7p15.2) at HOXA cluster. Interestingly, another miRNA involved in the HOX cluster, miR-10b, appears strongly upregulated in glioma. Recent reports have also indicated clinical implications of HOX genes in glioblastoma [30, 31], so the implications of miRNA dysregulations in HOX clusters should be clarified for glioblastomas. The function of miR196 is, however, more complicated, since the targets of miRNA usually number more than one hundred. Another target of miR196a, Annexin A1 (ANXA1), has also been implicated in glioblastoma as a mediator of apoptosis and an inhibitor of cell proliferation [32]. Previous study, which 
TABLE 1: Dysregulated miRNAs in glioblastoma.

\begin{tabular}{|c|c|c|c|c|c|c|c|}
\hline Authors & Year & Reference & Methods & Number of miRNA & Comparison & Upregulation & Downregulation \\
\hline \multirow{10}{*}{ Guan et al. } & \multirow{10}{*}{2010} & \multirow{10}{*}[19]{} & \multirow{10}{*}{ TaqMan array } & \multirow{10}{*}{365} & \multirow{10}{*}{ GBM versus $A A$} & miR-15b & miR-105 \\
\hline & & & & & & miR-21 & miR-128-2 \\
\hline & & & & & & miR-135(b) & miR-184 \\
\hline & & & & & & miR-196a & miR-302b \\
\hline & & & & & & miR-196b & miR-302d \\
\hline & & & & & & miR-363 & miR-367 \\
\hline & & & & & & & miR-383 \\
\hline & & & & & & & miR-504 \\
\hline & & & & & & & $\operatorname{miR}-517 \mathrm{c}$ \\
\hline & & & & & & & miR-601 \\
\hline \multirow{9}{*}{ Ciafrè et al. } & \multirow{9}{*}{2005} & \multirow{9}{*}[13]{} & \multirow{9}{*}{ Microarray } & \multirow{9}{*}{245} & \multirow{9}{*}{ GBM versus NB } & $\operatorname{miR}-9$ & miR-128-1 \\
\hline & & & & & & miR-10b & miR-181a \\
\hline & & & & & & miR-21 & miR-181b \\
\hline & & & & & & miR-25 & miR-181c \\
\hline & & & & & & miR-123 & \\
\hline & & & & & & miR-125b-1 & \\
\hline & & & & & & miR-125b-2 & \\
\hline & & & & & & miR-130a & \\
\hline & & & & & & $\operatorname{miR}-221$ & \\
\hline \multirow{5}{*}{ Chan et al. } & \multirow{5}{*}{2005} & \multirow{5}{*}[12]{} & \multirow{5}{*}{ Microarray } & \multirow{5}{*}{180} & \multirow{5}{*}{ GBM versus NB } & miR-21 & miR-188 \\
\hline & & & & & & miR-135(b) & miR-198 \\
\hline & & & & & & miR-138 & miR-202 \\
\hline & & & & & & miR-291-5' & \\
\hline & & & & & & miR-347 & \\
\hline \multirow{11}{*}{ Godlewski et al. } & & & & & & miR-10b & miR-124a \\
\hline & & & & & & miR-21 & miR-128-1 \\
\hline & & & & & & $\operatorname{miR}-26$ & miR-128-2 \\
\hline & & & & & & miR-383 & miR-137 \\
\hline & & & & & & miR-451 & miR-139 \\
\hline & 2008 & {$[15]$} & Microarray & 161 & GBM versus ANB & miR-486 & miR-190 \\
\hline & & & & & & miR-516-35p & miR-218 \\
\hline & & & & & & miR-519d & miR-299 \\
\hline & & & & & & & miR-323 \\
\hline & & & & & & & $\operatorname{miR}-483$ \\
\hline & & & & & & & miR-511-1 \\
\hline & & & & & & miR-10b & miR-134 \\
\hline & & & & & & miR-21 & miR-302c \\
\hline Sasayama et al. & 2009 & {$[16]$} & Microarray & 127 or 188 & GBM versus $A N B$ & miR-92b & miR-329 \\
\hline & & & & & & miR-106b & miR-369-3p \\
\hline & & & & & & miR-183 & miR-379 \\
\hline & & & & & & $\operatorname{miR}-9$ & miR-184 \\
\hline & & & & & & miR-15a & miR-328 \\
\hline & & & & & & miR-16 & \\
\hline & & & & & & miR-17 & \\
\hline & & & & & & miR-19a & \\
\hline Malzkorn et al & 2010 & {$[20]$} & TagMan & 157 & GBM versus DA & miR-20a & \\
\hline & & & & & & miR-21 & \\
\hline & & & & & & miR-25 & \\
\hline & & & & & & $\operatorname{miR}-28$ & \\
\hline & & & & & & miR-130b & \\
\hline & & & & & & miR-140 & \\
\hline & & & & & & miR-210 & \\
\hline
\end{tabular}


TABle 1: Continued.

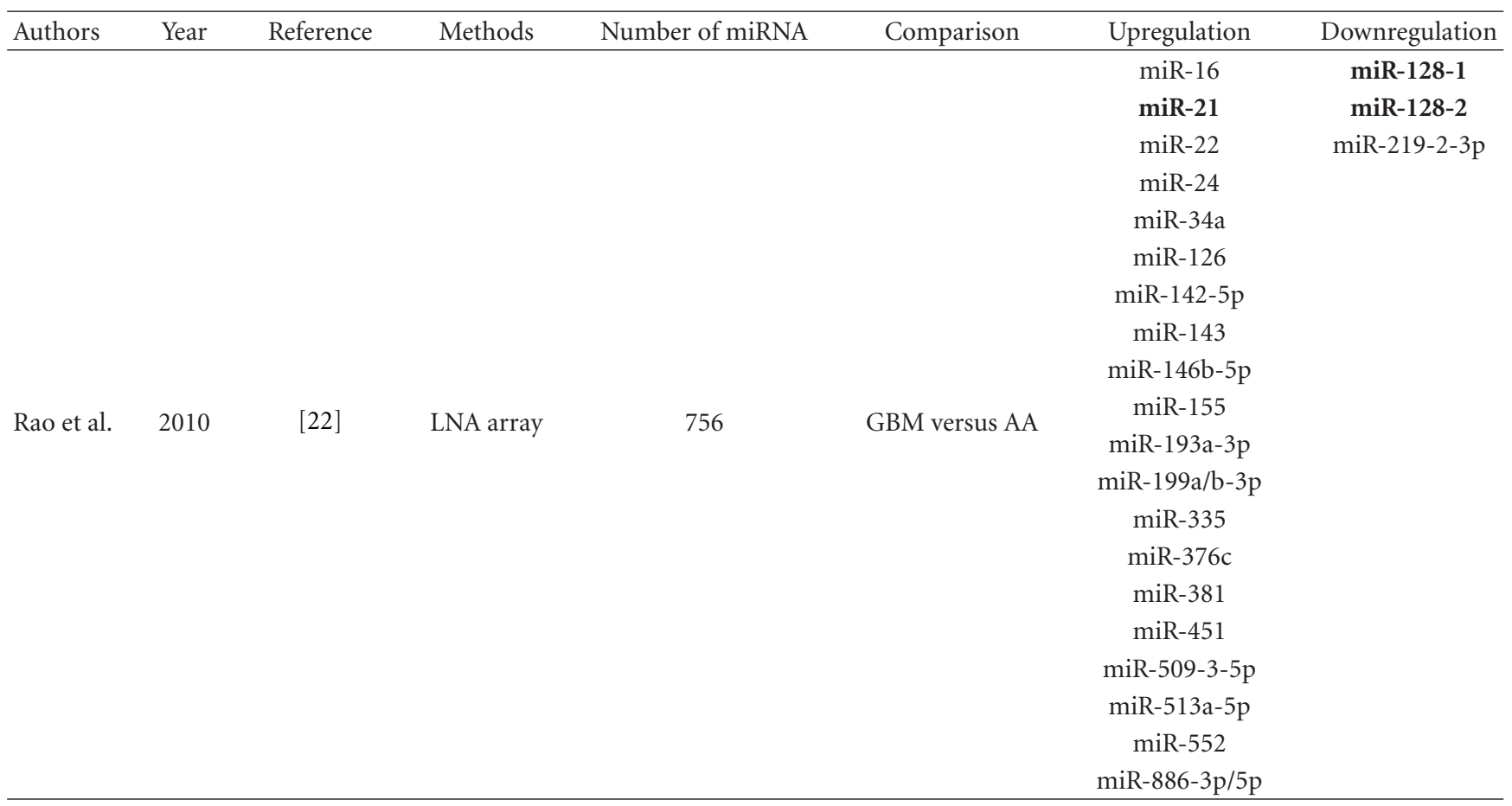

GBM: glioblastoma, AA: anaplastic astrocytoma, NB: normal brain, ANB: adjacent normal brain, DA: diffuse astrocytoma, Bold: common dysregulated miRNA (more than 3 reports).

evaluated miRNA expression in human cancer cell lines, also showed overexpression of miR-196 in glioblastoma cell lines [27]. However, Lakomy et al. reported an association opposite to our finding between miR-196b expression and clinical outcome, indicating another important aspects [33]. Further investigation is necessary to elucidate the clinical implications and biological functions of miR-196 in malignant gliomas.

\section{5. miR-10b}

Upregulation of miR-10b is one of the common aberrations in glioblastoma, which is identified by several recent reports $[13,15,16,24,34]$ (Table 1). Importantly, miR-10b is overexpressed in the vast majority of glioblastoma, whereas it is not detected in normal brain. Previous reports showed that miR-10b was overexpressed in breast cancer and regulated invasion and metastasis by targeting $\operatorname{HOXD} 10[35,36]$. Sasayama et al. reported that the expression of RhoC and UPAR was correlated with miR-10b expression level and lead to multifocal and dissemination of glioblastoma [16]. They indicated that miR-10b was associated with the invasion and migration of glioma cells [16]. However, Gabriely et al. demonstrated that in glioblastoma miR-10b regulated cell cycle and programmed cell death via regulation of Bim, TFAP2C, p16, and p21 but not HOXD10 and was significantly associated with patients' survival [34]. In glioblastoma, the function of miR-10b appeared to be different from breast cancer. However, it remains under investigation whether cellular environments affect the function of miR$10 \mathrm{~b}$.

\section{6. $\operatorname{miR}-128$}

Godlewski et al. identified downregulation of miR-128 in glioblastoma compared with adjacent brain, leading to a reduction in self-renewal of glioma stem cells via Bmi1 downregulation [15]. That report was the first demonstration of an association between miRNA and stem-cell properties in glioma. In addition, TCGA revealed that miR128 expression is lower in high-grade gliomas than in lowgrade gliomas. A recent study revealed that reduced miR-128 levels are associated with dedifferentiation and aggressiveness of malignant gliomas via EGFR/PDGF/AKT signaling [37]. Interestingly, miR-128 is enriched in brain, and associated with terminally differentiated neuron $[38,39]$. Verhaak et al. have revealed that miR-128 was repressed in glioblastoma classified the aggressive/dedifferentiated tumor subtype [2]. MiR-128 has been shown to be able to repress the growth of glioma initiating cell in vivo [37]. These findings support that miR-128 has a potential to achieve its therapeutic effect by suppressing proliferation and enhancing differentiation of glioma initiating cells.

\section{Subclassification Based on miRNA Expression Profiles}

Two classifications based on mRNA expression profiles have been reported in recent year, and both identified one of the subclasses as showing an expression signature resembling that of a "proneural" precursor cell $[1,2]$. However, the other subclasses of glioblastomas have shown little association with 
neural differentiation, and disagreement remains regarding the relationship of the subclasses to clinical outcomes $[1,2]$. Establishment of more accurate diagnostic and therapeutic strategies based on pathogenetic subclassification is obviously clinically important.

Recent attempts at miRNA expression profiling may show the way toward more accurate classifications of human cancers than mRNA expression profiling [8]. Furthermore, the recent large-scale multidimensional analysis of molecular characteristics, TCGA, which includes expression profiles of miRNA along with DNA copy number, gene expression and DNA methylation, has revealed frequent genetic alterations in three critical core pathways [9]. In fact, Kim et al. identified five clusters of glioblastoma based on miRNA expression profiles, which appeared to predict clinical outcomes more precisely than mRNA profiles [24]. Expression profiles using miRNA are useful for subclassification of glioblastoma and could identify novel therapeutic targets.

\section{Future Directions}

Since the introduction of temozolomide (TMZ) as standard chemotherapy, concomitant TMZ and radiotherapy have improved both progression-free survival and overall survival in patients with GBM [40]. However, the clinical prognosis of patients with GBM remains poor, with a median overall survival of only 14.6 months [40]. The cancer stem cell (CSC) hypothesis, that tumors are driven by a subpopulation of tumor cells with stem cell-like properties, may provide new insights into the radio- and chemo-resistance of GBM. Based on this hypothesis, some therapies in which shrink tumors show reduced diameter might not be associated with improved rates of cure if CSCs fail to be eliminated, and the therapies targeting CSCs should prove clinically more effective [41]. Elucidating the molecular biology of CSCs would thus be crucial.

Recently, some miRNAs have been reported to contribute to CSC properties and cancer heterogeneity. In addition to miR-128 described above, miR-124, -137 [42], -34a $[43,44]$, and -326 [45] have been reported to play roles in the maintenance of CSC properties. The identification of miRNAs thus adds another layer of gene expression associated with gliomagenesis and maintenance of CSC properties via regulation of targeted signaling pathways. The regulation of aberrant miRNAs could affect not only conventional radio- and chemo-therapy, but also the sensitivity to molecular target therapy. Further study of miRNAs function in CSC may lead to novel treatment strategies for GBM patients. Consideration of the therapeutic application of miRNAs suggests two putative options: inhibition of oncogenic miRNAs and replacement of tumor-suppressor miRNAs. Development of the ideal delivery systems to target tissue remains under investigation. However, miRNA-based therapy could be more practical than gene therapy, since miRNA is a substantially small molecule.

In conclusion, miRNA appears to offer a crucial biomarker not only as a diagnostic marker, but also as a target for molecular therapies. The miRNA approach to therapy could thus achieve modest changes for multiple genes and may prove more effective than current molecular targeted therapies to target single gene within an oncogenic signaling pathway. Molecular-targeted therapy based on miRNA expression in CSCs has the potential to allow more personalized and effective treatment strategies in the nottoo-distant future.

\section{Conflict of Interests}

There is no potential conflict of interests to be disclosed.

\section{Acknowledgment}

This work was supported by grants from the Ministry of Education, Culture, Sports, Science and Technology (MEXT) of Japan; Grant numbers: 2390280 and 3659691 (M. Mizoguchi).

\section{References}

[1] H. S. Phillips, S. Kharbanda, R. Chen et al., "Molecular subclasses of high-grade glioma predict prognosis, delineate a pattern of disease progression, and resemble stages in neurogenesis," Cancer Cell, vol. 9, no. 3, pp. 157-173, 2006.

[2] R. G. W. Verhaak, K. A. Hoadley, E. Purdom et al., "Integrated genomic analysis identifies clinically relevant subtypes of glioblastoma characterized by abnormalities in PDGFRA, IDH1, EGFR, and NF1," Cancer Cell, vol. 17, no. 1, pp. 98$110,2010$.

[3] J. T. Huse, H. S. Phillips, and C. W. Brennan, "Molecular subclassification of diffuse gliomas: seeing order in the chaos," Glia, vol. 59, no. 8, pp. 1190-1199, 2011.

[4] D. P. Bartel, "MicroRNAs: genomics, biogenesis, mechanism, and function," Cell, vol. 116, no. 2, pp. 281-297, 2004.

[5] V. Ambros, "The functions of animal microRNAs," Nature, vol. 431, no. 7006, pp. 350-355, 2004.

[6] A. Kozomara and S. Griffiths-Jones, "MiRBase: integrating microRNA annotation and deep-sequencing data," Nucleic Acids Research, vol. 39, no. 1, pp. D152-D157, 2011.

[7] W. Filipowicz, S. N. Bhattacharyya, and N. Sonenberg, "Mechanisms of post-transcriptional regulation by microRNAs: are the answers in sight?" Nature Reviews Genetics, vol. 9, no. 2, pp. 102-114, 2008.

[8] J. Lu, G. Getz, E. A. Miska et al., "MicroRNA expression profiles classify human cancers," Nature, vol. 435, no. 7043, pp. 834-838, 2005.

[9] Network TCGAr, "Comprehensive genomic characterization defines human glioblastoma genes and core pathways," Nature, vol. 455, no. 7216, pp. 1061-1068, 2008.

[10] G. A. Calin, "Frequent deletions and down-regulation of micro-RNA genes miR15 and miR16 at 13q14 in chronic lymphocytic leukemia," Proceedings of the National Academy of Sciences of the United States of America, vol. 99, no. 24, pp. 15524-15529, 2002.

[11] G. A. Calin, "Human microRNA genes are frequently located at fragile sites and genomic regions involved in cancers," Proceedings of the National Academy of Sciences of the United States of America, vol. 101, no. 9, pp. 2999-3004, 2004.

[12] J. A. Chan, A. M. Krichevsky, and K. S. Kosik, "MicroRNA-21 is an antiapoptotic factor in human glioblastoma cells," Cancer Research, vol. 65, no. 14, pp. 6029-6033, 2005. 
[13] S. A. Ciafrè, S. Galardi, A. Mangiola et al., "Extensive modulation of a set of microRNAs in primary glioblastoma," Biochemical and Biophysical Research Communications, vol. 334, no. 4, pp. 1351-1358, 2005.

[14] T. Papagiannakopoulos, A. Shapiro, and K. S. Kosik, "MicroRNA-21 targets a network of key tumor-suppressive pathways in glioblastoma cells," Cancer Research, vol. 68, no. 19, pp. 8164-8172, 2008.

[15] J. Godlewski, M. O. Nowicki, A. Bronisz et al., "Targeting of the Bmi-1 oncogene/stem cell renewal factor by MicroRNA128 inhibits glioma proliferation and self-renewal," Cancer Research, vol. 68, no. 22, pp. 9125-9130, 2008.

[16] T. Sasayama, M. Nishihara, T. Kondoh, K. Hosoda, and E. Kohmura, "MicroRNA-10b is overexpressed in malignant glioma and associated with tumor invasive factors, UPAR and RhoC," International Journal of Cancer, vol. 125, no. 6, pp. 1407-1413, 2009.

[17] Y. Chen, J. A. L. Gelfond, L. M. McManus, and P. K. Shireman, "Reproducibility of quantitative RT-PCR array in miRNA expression profiling and comparison with microarray analysis," BMC Genomics, vol. 10, article 407, 2009.

[18] P. Mestdagh, P. Van Vlierberghe, A. De Weer et al., "A novel and universal method for microRNA RT-qPCR data normalization," Genome Biology, vol. 10, no. 6, article R64, 2009.

[19] Y. Guan, M. Mizoguchi, K. Yoshimoto et al., "MiRNA-196 is upregulated in glioblastoma but not in anaplastic astrocytoma and has prognostic significance," Clinical Cancer Research, vol. 16, no. 16, pp. 4289-4297, 2010.

[20] B. Malzkorn, M. Wolter, F. Liesenberg et al., "Identification and functional characterization of microRNAs involved in the malignant progression of gliomas," Brain Pathology, vol. 20, no. 3, pp. 539-550, 2010.

[21] H. Kim, W. Huang, X. Jiang, B. Pennicooke, P. J. Park, and M. D. Johnson, "Integrative genome analysis reveals an oncomir/oncogene cluster regulating glioblastoma survivorship," Proceedings of the National Academy of Sciences of the United States of America, vol. 107, no. 5, pp. 2183-2188, 2010.

[22] S. A. M. Rao, V. Santosh, and K. Somasundaram, "Genomewide expression profiling identifies deregulated miRNAs in malignant astrocytoma," Modern Pathology, vol. 23, no. 10, pp. 1404-1417, 2010.

[23] S. Srinivasan, I. R. P. Patric, and K. Somasundaram, "A TenmicroRNA expression signature predicts survival in glioblastoma," PLoS ONE, vol. 6, no. 3, Article ID e17438, 2011.

[24] T. M. Kim, W. Huang, R. Park, P. J. Park, and M. D. Johnson, "A developmental taxonomy of glioblastoma defined and maintained by microRNAs," Cancer Research, vol. 71, no. 9, pp. 3387-3399, 2011.

[25] P. P. Medina, M. Nolde, and F. J. Slack, "OncomiR addiction in an in vivo model of microRNA-21-induced pre-B-cell lymphoma," Nature, vol. 467, no. 7311, pp. 86-90, 2010.

[26] S. Volinia, G. A. Calin, C. G. Liu et al., "A microRNA expression signature of human solid tumors defines cancer gene targets," Proceedings of the National Academy of Sciences of the United States of America, vol. 103, no. 7, pp. 2257-2261, 2006.

[27] A. Gaur, D. A. Jewell, Y. Liang et al., "Characterization of microRNA expression levels and their biological correlates in human cancer cell lines," Cancer Research, vol. 67, no. 6, pp. 2456-2468, 2007.
[28] M. Bloomston, W. L. Frankel, F. Petrocca et al., "MicroRNA expression patterns to differentiate pancreatic adenocarcinoma from normal pancreas and chronic pancreatitis," Journal of the American Medical Association, vol. 297, no. 17, pp. 19011908, 2007.

[29] S. Yekta, I. H. Shih, and D. P. Bartel, "MicroRNA-directed cleavage of HOXB8 mRNA," Science, vol. 304, no. 5670, pp. 594-596, 2004.

[30] A. Murat, E. Migliavacca, T. Gorlia et al., "Stem cell-related "self-renewal" signature and high epidermal growth factor receptor expression associated with resistance to concomitant chemoradiotherapy in glioblastoma," Journal of Clinical Oncology, vol. 26, no. 18, pp. 3015-3024, 2008.

[31] N. Gaspar, L. Marshall, L. Perryman et al., "MGMTindependent temozolomide resistance in pediatric glioblastoma cells associated with a PI3-kinase-mediated HOX/stem cell gene signature," Cancer Research, vol. 70, no. 22, pp. 92439252, 2010.

[32] R. Luthra, R. R. Singh, M. G. Luthra et al., "MicroRNA196a targets annexin A1: a microRNA-mediated mechanism of annexin A1 downregulation in cancers," Oncogene, vol. 27, no. 52, pp. 6667-6678, 2008.

[33] R. Lakomy, J. Sana, S. Hankeova et al., "MiR-195, miR-196b, miR-181c, miR-21 expression levels and O-6-methylguanineDNA methyltransferase methylation status are associated with clinical outcome in glioblastoma patients," Cancer Science, vol. 102, no. 12, pp. 2186-2190, 2011.

[34] G. Gabriely, M. Yi, R. S. Narayan et al., "Human glioma growth is controlled by microRNA-10b," Cancer Research, vol. 71, no. 10, pp. 3563-3572, 2011.

[35] L. Ma, J. Teruya-Feldstein, and R. A. Weinberg, "Tumour invasion and metastasis initiated by microRNA-10b in breast cancer," Nature, vol. 449, no. 7163, pp. 682-688, 2007.

[36] C. H. Moriarty, B. Pursell, and A. M. Mercurio, "miR-10b targets Tiam1: implications for Rac activation and carcinoma migration," Journal of Biological Chemistry, vol. 285, no. 27, pp. 20541-20546, 2010.

[37] T. Papagiannakopoulos, D. Friedmann-Morvinski, P. Neveu et al., "Pro-neural miR-128 is a glioma tumor suppressor that targets mitogenic kinases," Oncogene, vol. 31, no. 15, pp. 18841895, 2012.

[38] A. M. Krichevsky, "A microRNA array reveals extensive regulation of microRNAs during brain development," RNA, vol. 9, no. 10, pp. 1274-1281, 2003.

[39] K. S. Kosik and A. M. Krichevsky, "The elegance of the microRNAs: a neuronal perspective," Neuron, vol. 47, no. 6, pp. 779-782, 2005.

[40] R. Stupp, W. P. Mason, M. J. van den Bent et al., "Radiotherapy plus concomitant and adjuvant temozolomide for glioblastoma," The New England Journal of Medicine, vol. 352, no. 10, pp. 987-996, 2005.

[41] T. Reya, S. J. Morrison, M. F. Clarke, and I. L. Weissman, "Stem cells, cancer, and cancer stem cells," Nature, vol. 414, no. 6859, pp. 105-111, 2001.

[42] J. Silber, D. A. Lim, C. Petritsch et al., "miR-124 and miR137 inhibit proliferation of glioblastoma multiforme cells and induce differentiation of brain tumor stem cells," BMC Medicine, vol. 6, article 14, 2008.

[43] L. Yunqing, F. Guessous, Z. Ying et al., "MicroRNA-34a inhibits glioblastoma growth by targeting multiple oncogenes," Cancer Research, vol. 69, no. 19, pp. 7569-7576, 2009. 
[44] F. Guessous, Y. Zhang, A. Kofman et al., "microRNA-34a is tumor suppressive in brain tumors and glioma stem cells," Cell Cycle, vol. 9, no. 6, pp. 1031-1036, 2010.

[45] B. Kefas, L. Comeau, D. H. Floyd et al., "The neuronal microRNA miR-326 acts in a feedback loop with notch and has therapeutic potential against brain tumors," Journal of Neuroscience, vol. 29, no. 48, pp. 15161-15168, 2009. 


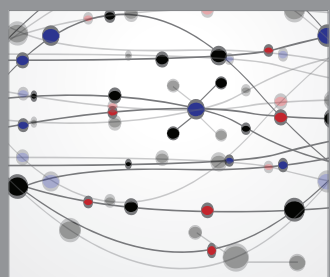

The Scientific World Journal
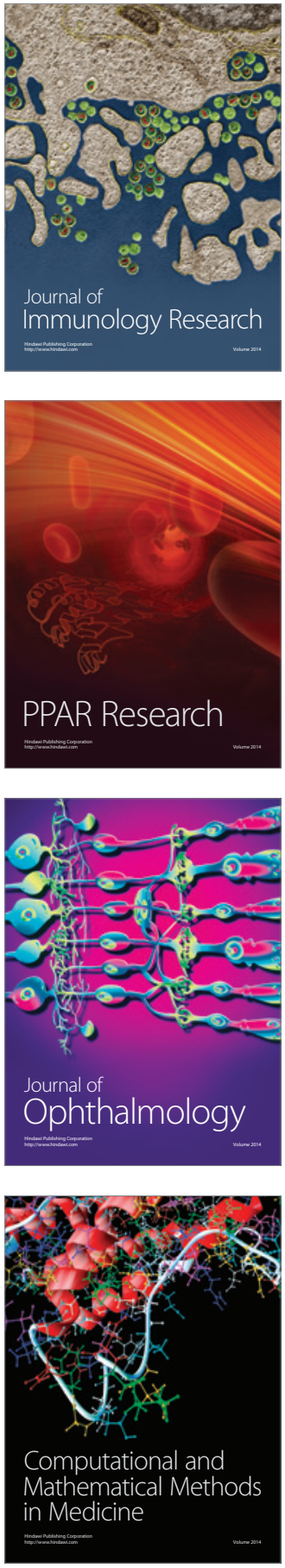

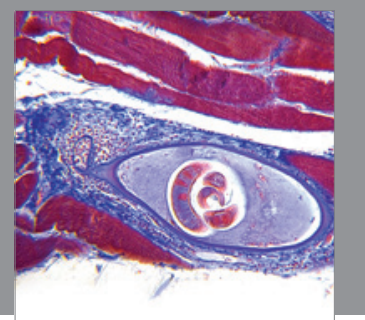

Gastroenterology

Research and Practice
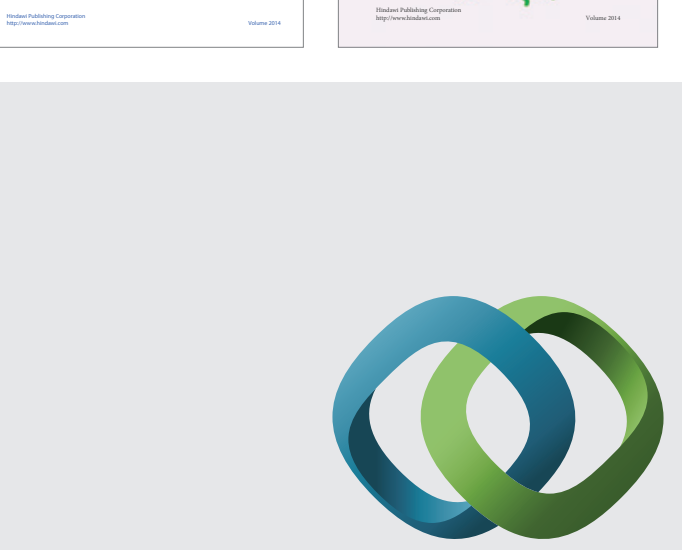

\section{Hindawi}

Submit your manuscripts at

http://www.hindawi.com
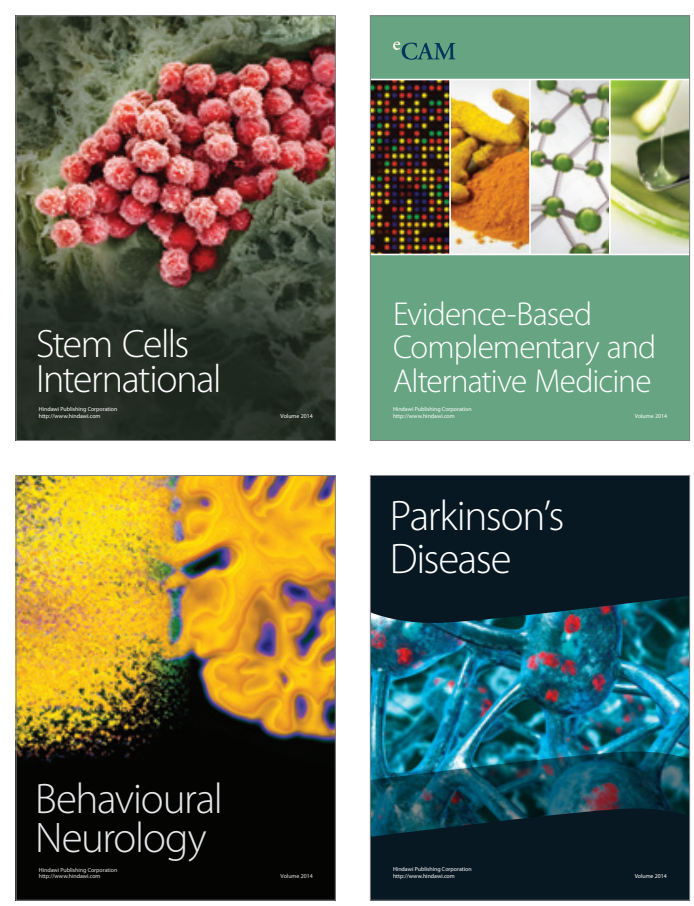

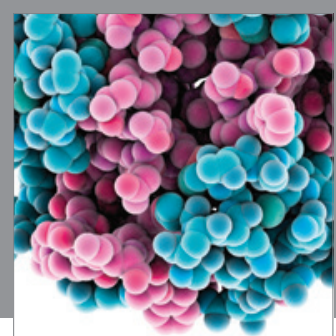

Journal of
Diabetes Research

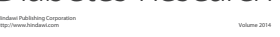

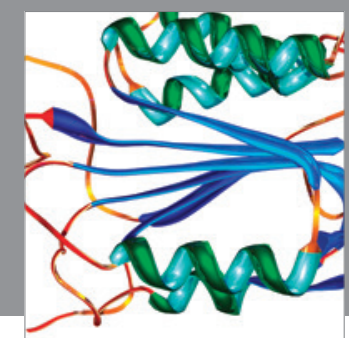

Disease Markers
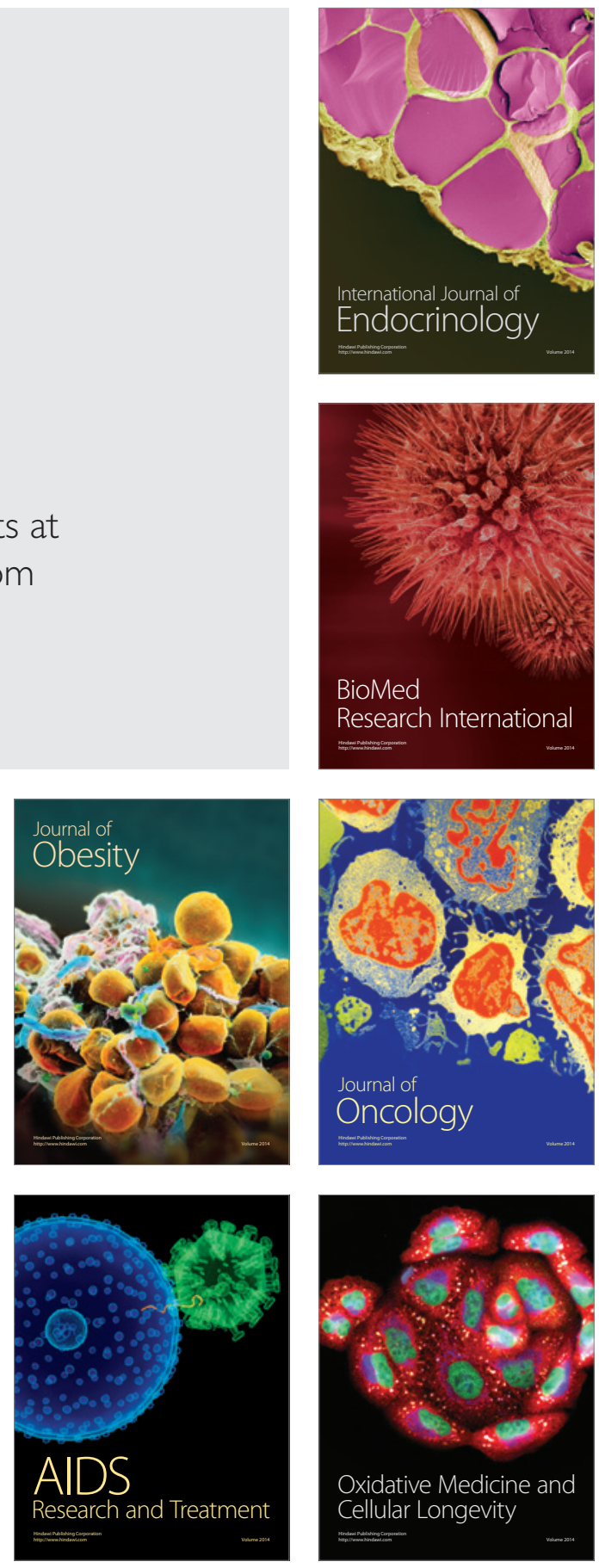